\title{
Generalized shift-invariant systems and approximately dual frames
}

Benavente, Ana; Christensen, Ole; Zakowicz, Maria I.

Published in:

Annals of Functional Analysis

Link to article, DOI:

$10.1215 / 20088752-3784315$

Publication date:

2017

Document Version

Peer reviewed version

Link back to DTU Orbit

Citation (APA):

Benavente, A., Christensen, O., \& Zakowicz, M. I. (2017). Generalized shift-invariant systems and approximately dual frames. Annals of Functional Analysis, 8(2), 177-189. https://doi.org/10.1215/20088752-3784315

\section{General rights}

Copyright and moral rights for the publications made accessible in the public portal are retained by the authors and/or other copyright owners and it is a condition of accessing publications that users recognise and abide by the legal requirements associated with these rights.

- Users may download and print one copy of any publication from the public portal for the purpose of private study or research.

- You may not further distribute the material or use it for any profit-making activity or commercial gain

- You may freely distribute the URL identifying the publication in the public portal

If you believe that this document breaches copyright please contact us providing details, and we will remove access to the work immediately and investigate your claim. 


\title{
GENERALIZED SHIFT-INVARIANT SYSTEMS AND APPROXIMATELY DUAL FRAMES
}

\author{
ANA BENAVENTE ${ }^{1}$, OLE CHRISTENSEN ${ }^{2}$ and MARÍA I. ZAKOWICZ ${ }^{3 *}$ \\ ABstract. Dual pairs of frames yield a procedure for obtaining perfect recon- \\ struction of elements in the underlying Hilbert space in terms of superpositions \\ of the frame elements. However, practical constraints often force us to apply \\ sequences that do not exactly form dual frames. In this paper we consider \\ the important case of generalized shift-invariant systems and provide various \\ ways of estimating the deviation from perfect reconstruction that occur when \\ the systems do not form dual frames. The deviation from being dual frames \\ will be measured either in terms of a perturbation condition or in terms of the \\ deviation from equality in the duality conditions.
}

\section{INTRODUCTION}

Frame theory is a tool to obtain expansions of elements in a Hilbert space in terms of "convenient building blocks." In fact, if two sequences $\left\{f_{k}\right\}_{k=1}^{\infty}$ and $\left\{g_{k}\right\}_{k=1}^{\infty}$ in a separable Hilbert space $\mathcal{H}$ form a pair of dual frames for $\mathcal{H}$, each $f \in \mathcal{H}$ has a representation

$$
f=\sum_{k=1}^{\infty}\left\langle f, g_{k}\right\rangle f_{k}
$$

In signal processing terms this is expressed by saying that dual pairs of frames leads to perfect reconstruction. However, practical constraints will often force us to deal with systems that do not lead to perfect reconstruction, e.g., sequences $\left\{f_{k}\right\}_{k=1}^{\infty}$ and $\left\{g_{k}\right\}_{k=1}^{\infty}$ that are not exactly dual frames. The purpose of this paper is to derive estimates for the corresponding deviation from equality in (1.1) in the important case where $\left\{f_{k}\right\}_{k=1}^{\infty}$ and $\left\{g_{k}\right\}_{k=1}^{\infty}$ are generalized shift-invariant systems in $L^{2}(\mathbb{R})$. The estimate will be formulated in terms of the operator norm

$$
\left\|I-\sum_{k=1}^{\infty}\left\langle\cdot, g_{k}\right\rangle f_{k}\right\|=\sup _{\|f\|=1}\left\|f-\sum_{k=1}^{\infty}\left\langle f, g_{k}\right\rangle f_{k}\right\| ;
$$

and the deviation from $\left\{f_{k}\right\}_{k=1}^{\infty}$ and $\left\{g_{k}\right\}_{k=1}^{\infty}$ being dual frames will be measured in terms of a perturbation condition or in terms of the deviation form the duality conditions (see Section 2 for details).

Copyright 2016 by the Tusi Mathematical Research Group.

Date: Received: xxxxxx; Revised: yyyyyy; Accepted: zzzzzz.

${ }^{2}$ Corresponding author.

2010 Mathematics Subject Classification. Primary 42C15; Secondary 46E40.

Key words and phrases. frames, approximately dual frames, generalized shift-invariant systems. 
The paper is organized as follows. In the rest of the introduction we provide the necessary information about frame theory and approximately dual frames. Section 2 discusses the generalized shift-invariant systems. The new results concerning approximate dual frames are stated and proved in Section 3.

In order to set the stage for the discussion to follow, recall that a sequence $\left\{f_{k}\right\}_{k=1}^{\infty}$ in a Hilbert space $\mathcal{H}$ is a frame for $\mathcal{H}$ if there exist constants $A, B>0$ such that

$$
A\|f\|^{2} \leq \sum_{k=1}^{\infty}\left|\left\langle f, f_{k}\right\rangle\right|^{2} \leq B\|f\|^{2}, \forall f \in \mathcal{H} .
$$

The sequence $\left\{f_{k}\right\}_{k=1}^{\infty}$ is a Bessel sequence if at least the upper condition in (1.2) holds; and two frames $\left\{f_{k}\right\}_{k=1}^{\infty}$ and $\left\{g_{k}\right\}_{k=1}^{\infty}$ are dual frames if (1.1) holds. Note that if $\left\{f_{k}\right\}_{k=1}^{\infty}$ and $\left\{g_{k}\right\}_{k=1}^{\infty}$ are Bessel sequences and (1.1) holds, then $\left\{f_{k}\right\}_{k=1}^{\infty}$ and $\left\{g_{k}\right\}_{k=1}^{\infty}$ are automatically frames.

Given any Bessel sequence $\left\{f_{k}\right\}_{k=1}^{\infty}$ one can define a bounded operator $T$ : $\ell^{2}(\mathbb{N}) \rightarrow \mathcal{H}$ by $T\left\{c_{k}\right\}_{k=1}^{\infty}:=\sum c_{k} f_{k}$; the operator $T$ is called the synthesis operator or preframe operator. It is easy to see that the adjoint operator is given by $T^{*}: \mathcal{H} \rightarrow \ell^{2}(\mathbb{N}), T^{*} f=\left\{\left\langle f, f_{k}\right\rangle\right\}_{k=1}^{\infty}$. Denoting the synthesis operators for two Bessel sequences $\left\{f_{k}\right\}_{k=1}^{\infty}$ and $\left\{g_{k}\right\}_{k=1}^{\infty}$ by $T$, respectively, $U$, it is clear that $\left\{f_{k}\right\}_{k=1}^{\infty}$ and $\left\{g_{k}\right\}_{k=1}^{\infty}$ are dual frames if and only if

$$
T U^{*}=I \text {. }
$$

For more information on frames we refer to $[6,7,1]$. In case $\left\{f_{k}\right\}_{k=1}^{\infty}$ and $\left\{g_{k}\right\}_{k=1}^{\infty}$ are not precisely a pair of dual frames it is important to be able to measure the deviation from perfect reconstruction; for example, it would be preferable to obtain an estimate of the form

$$
\left\|f-\sum_{k=1}^{\infty}\left\langle f, g_{k}\right\rangle f_{k}\right\| \leq \epsilon\|f\|, \forall f \in \mathcal{H}
$$

for a small value of $\epsilon>0$. For the case where $\left\{f_{k}\right\}_{k=1}^{\infty}$ and $\left\{g_{k}\right\}_{k=1}^{\infty}$ are Bessel sequences, this idea has been formalized in the paper [3] by saying that $\left\{f_{k}\right\}_{k=1}^{\infty}$ and $\left\{g_{k}\right\}_{k=1}^{\infty}$ form approximately dual frames if (1.4) holds for some $\epsilon<1$. Further applications appear in [9] by Feichtinger, Grybos and Onchis, and [10] by Feichtinger, Onchis and Wiesmeyr; the latter contains a discussion of wavelet frames and approximation on subspaces, however, not with the exact concept of approximately dual frames as defined in in [3]. The results in the current paper will provide various ways of estimating $\epsilon$ for the important class of generalized shift-invariant systems, to be introduced next.

\section{Preliminaries on GSI-Systems}

Generalized shift-invariant systems (GSI-systems) were introduced by Hernandez, Labate \& Weiss [13] and Ron \& Shen [20] as a general framework for considering Gabor systems, shift-invariant systems, and wavelet systems simultaneously. We return to a more detailed description of these systems in Section 3. 
Considering the translation operator

$$
T_{y}: L^{2}(\mathbb{R}) \rightarrow L^{2}(\mathbb{R}), T_{y} f(x)=f(x-y), x, y \in \mathbb{R},
$$

the formal definition of a GSI-system is as follows.

Definition 2.1. A generalized shift invariant system in $L^{2}(\mathbb{R})$ is a collection of functions of the form $\left\{T_{c_{j} k} \phi_{j}\right\}_{k \in \mathbb{Z}, j \in J}$, where $\left\{\phi_{j}\right\}_{j \in J} \subset L^{2}(\mathbb{R})$ and $\left\{c_{j}\right\}_{j \in J}$ is a countable collection of positive numbers.

For the purpose of this paper we need to be able to verify the Bessel condition for a GSI-system, and to characterize dual frames with the GSI-structure. In this section we will provide the necessary background information on this.

The following result from [4] provides a convenient sufficient condition for a GSI-system to be a Bessel sequence; it is a generalization of a result in [18]. It is formulated in terms of the Fourier transform, on $L^{1}(\mathbb{R})$ defined by $\widehat{f}(\gamma)=$ $\int_{-\infty}^{\infty} f(x) e^{-2 \pi i x \gamma} d x$, and extended $L^{2}(\mathbb{R})$ in the usual way.

Lemma 2.2. Given a GSI-system $\left\{T_{c_{j} k} \phi_{j}\right\}_{j \in J, k \in \mathbb{Z}}$ in $L^{2}(\mathbb{R})$, assume that

$$
B:=\sup _{\gamma \in \mathbb{R}} \sum_{j \in J} \sum_{k \in \mathbb{Z}} \frac{1}{c_{j}}\left|\widehat{\phi}_{j}(\gamma) \widehat{\phi}_{j}\left(\gamma-c_{j}^{-1} k\right)\right|<\infty .
$$

Then $\left\{T_{c_{j} k} \phi_{j}\right\}_{j \in J, k \in \mathbb{Z}}$ is a Bessel sequence with bound $B$.

For generalized shift-invariant systems, perfect reconstruction has been characterized in terms of a number of equations. In order to state the duality conditions we need certain technical conditions. Let

$$
\mathcal{D}:=\left\{f \in L^{2}(\mathbb{R}) \mid \operatorname{supp} \widehat{f} \text { is compact and } \widehat{f} \in L^{\infty}(\mathbb{R})\right\} .
$$

It is clear that $\mathcal{D}$ is a dense subspace of $L^{2}(\mathbb{R})$.

The duality conditions are valid under certain very mild technical conditions, stated next. The local integrability condition (LIC) was introduced by Hernandez, Labate, and Weiss [13]; the weaker $\alpha$-local integrability condition ( $\alpha$-LIC) appeared in [14] by Jakobsen and Lemvig.

Definition 2.3. Consider two GSI-systems $\left\{T_{c_{j} k} \phi_{j}\right\}_{k \in \mathbb{Z}, j \in J}$ and $\left\{T_{c_{j} k} \widetilde{\phi}_{j}\right\}_{k \in \mathbb{Z}, j \in J}$. (i) If

$$
\sum_{j \in \mathbb{Z}} \sum_{m \in \mathbb{Z}} \frac{1}{c_{j}} \int_{\text {supp } \widehat{f}}\left|\widehat{f}\left(\gamma+c_{j}^{-1} m\right) \widehat{\phi}_{j}(\gamma)\right|^{2} d \gamma<\infty
$$

for all $f \in \mathcal{D}$, we say that $\left\{T_{c_{j} k} \phi_{j}\right\}_{k \in \mathbb{Z}, j \in J}$ satisfies the LIC condition.

(ii) $\left\{T_{c_{j} k} \phi_{j}\right\}_{k \in \mathbb{Z}, j \in J}$ and $\left\{T_{c_{j} k} \widetilde{\phi}_{j}\right\}_{k \in \mathbb{Z}, j \in J}$ satisfy the dual $\alpha$-LIC condition if

$$
\sum_{j \in \mathbb{Z}} \sum_{m \in \mathbb{Z}} \frac{1}{c_{j}} \int_{-\infty}^{\infty}\left|\widehat{f}(\gamma) \widehat{f}\left(\gamma+c_{j}^{-1} m\right) \widehat{\phi}_{j}(\gamma) \widehat{\widetilde{\phi}}_{j}\left(\gamma+c_{j}^{-1} m\right)\right| d \gamma<\infty
$$

for all $f \in \mathcal{D}$.

We say that $\left\{T_{c_{j} k} \phi_{j}\right\}_{j \in \mathbb{Z}}$ satisfies the $\alpha$-LIC condition, if (2.3) holds with $\phi_{j}=\widetilde{\phi}_{j}$. 
Finally, in order to formulate the duality conditions we need to consider a certain re-indexing of the GSI-systems. Given a GSI-system $\left\{T_{c_{j} k} \phi_{j}\right\}_{k \in \mathbb{Z}, j \in J}$, let

$$
\Lambda:=\left\{c_{j}^{-1} n: j \in J, n \in \mathbb{Z}\right\}
$$

and, for $\alpha \in \Lambda$, let

$$
J_{\alpha}:=\left\{j \in J: \exists n \in \mathbb{Z} \text { such that } \alpha=c_{j}^{-1} n\right\} .
$$

In [13], Hernandez, Labate and Weiss characterized duality for two GSI-systems satisfying the LIC condition. Jakobsen and Lemvig proved in [14] that the same result holds under the weaker dual $\alpha$-LIC condition:

Proposition 2.4. Assume that the GSI-systems $\left\{T_{c_{j} k} \phi_{j}\right\}_{k \in \mathbb{Z}, j \in J}$ and $\left\{T_{c_{j} k} \widetilde{\phi}_{j}\right\}_{k \in \mathbb{Z}, j \in J}$ are Bessel sequences and satisfy the dual $\alpha$-LIC condition. Then $\left\{T_{c_{j} k} \phi_{j}\right\}_{k \in \mathbb{Z}, j \in J}$ and $\left\{T_{c_{j} k} \widetilde{\phi}_{j}\right\}_{k \in \mathbb{Z}, j \in J}$ are dual frames if and only if

$$
\sum_{j \in J_{\alpha}} \frac{1}{c_{j}} \overline{\widehat{\phi}_{j}(\gamma)} \widehat{\widetilde{\phi}}_{j}(\gamma+\alpha)=\delta_{\alpha, 0}, \text { a.e. } \gamma \in \mathbb{R}
$$

for all $\alpha \in \Lambda$.

Note that (2.6) is equivalent to the equations

$$
\left\{\begin{array}{l}
\sum_{j \in J} \frac{1}{c_{j}} \overline{\widehat{\phi}_{j}(\gamma)} \widehat{\widetilde{\phi}}_{j}(\gamma)-1=0 \\
\sum_{j \in J_{\alpha}} \frac{1}{c_{j}} \overline{\widehat{\phi}_{j}(\gamma)} \widehat{\widetilde{\phi}_{j}}(\gamma+\alpha)=0, \alpha \in \Lambda \backslash\{0\}
\end{array}\right.
$$

The formulation (2.7) is more convenient for our purpose. In fact, for GSIsystems $\left\{T_{c_{j} k} \phi_{j}\right\}_{k \in \mathbb{Z}, j \in J}$ and $\left\{T_{c_{j} k} \widetilde{\phi}_{j}\right\}_{k \in \mathbb{Z}, j \in J}$ we will show that we can estimate the deviation from perfect reconstruction by the deviation from equality in (2.7).

\section{Approximately dual GSI-frames}

We will now derive various ways of estimating the deviation from perfect reconstruction for a pair of GSI-systems. The first result (to be stated in Theorem 3.3) will measure the deviation from the given systems being dual frames directly in terms of the deviation from equality in the duality conditions (2.7). We begin with a few technical lemma's.

Lemma 3.1. Assume that $\left\{T_{c_{j} k} \phi_{j}\right\}_{k \in \mathbb{Z}, j \in J}$ and $\left\{T_{c_{j} k} \widetilde{\phi}_{j}\right\}_{k \in \mathbb{Z}, j \in J}$ satisfy the dual $\alpha$-LIC condition. Then

$$
\sum_{j \in \mathbb{Z}} \sum_{m \in \mathbb{Z}} \frac{1}{c_{j}} \int_{-\infty}^{\infty}\left|\widehat{f}(\gamma) \widehat{g}\left(\gamma+c_{j}^{-1} m\right) \widehat{\phi}_{j}(\gamma) \widehat{\widetilde{\phi}}_{j}\left(\gamma+c_{j}^{-1} m\right)\right| d \gamma<\infty,
$$

for all $f, g \in \mathcal{D}$.

Proof. Define the function $\kappa$ via $\widehat{\kappa}(\gamma)=\max (|\widehat{f}(\gamma)|,|\widehat{g}(\gamma)|)$; then $\kappa \in \mathcal{D}$ and

$$
\left|\widehat{f}(\gamma) \widehat{g}\left(\gamma+c_{j}^{-1} m\right)\right| \leq\left|\widehat{\kappa}(\gamma) \widehat{\kappa}\left(\gamma+c_{j}^{-1} m\right)\right| .
$$


Thus, the expression (3.1) is finite by the dual $\alpha$-LIC condition applied on the function $\kappa$.

The following lemma is a variant of a result in [13], which is a key step in the proof of Proposition 2.4. The modifications consist in the use of the dual $\alpha$-LIC condition instead of the stronger LIC-condition used in [13]; also, in [13] the functions $f$ and $g$ below were identical, while it is essential for our purpose that they are allowed to be different functions.

Lemma 3.2. Assume that the GSI-systems $\left\{T_{c_{j} k} \phi_{j}\right\}_{k \in \mathbb{Z}, j \in J}$ and $\left\{T_{c_{j} k} \widetilde{\phi}_{j}\right\}_{k \in \mathbb{Z}, j \in J}$ satisfy the dual $\alpha$-LIC-condition. Then for $f, g \in \mathcal{D}$, the function

$$
\omega(y):=\sum_{j \in J} \sum_{k \in \mathbb{Z}}\left\langle T_{y} f, T_{c_{j} k} \phi_{j}\right\rangle\left\langle T_{c_{j} k} \widetilde{\phi}_{j}, T_{y} g\right\rangle
$$

is continuous, and

$$
\omega(y)=\sum_{\alpha \in \Lambda}\left(\int_{-\infty}^{\infty} \widehat{f}(\gamma) \overline{\widehat{g}(\gamma+\alpha)} \sum_{j \in J_{\alpha}} \frac{1}{c_{j}} \overline{\widehat{\phi}_{j}(\gamma)} \widehat{\widetilde{\phi}}_{j}(\gamma+\alpha)\right) e^{2 \pi i \alpha \cdot y}
$$

pointwise for all $y \in \mathbb{R}$.

Proof. We will refer to [13] for the parts of the proof that are unaffected by the mentioned changes, and focus on the parts where the dual $\alpha$-LIC condition is used. First, for any $f \in \mathcal{D}$, the arguments in [13] show that $\left\langle f, T_{c_{j} k} \phi_{j}\right\rangle$ is the $(-k)$ th Fourier coefficient of the 1-periodic function

$$
F_{j}(\mu)=\frac{1}{c_{j}} \sum_{n \in \mathbb{Z}} \widehat{f}\left(c_{j}^{-1}(\mu+n)\right) \overline{\widehat{\phi}_{j}\left(c_{j}^{-1}(\mu+n)\right)} .
$$

Using Parseval's equation and elementary manipulations on the sums (see [13] or [1]) it follows that for $j \in J$, the function

$$
\omega_{j}(y):=\sum_{k \in \mathbb{Z}}\left\langle T_{y} f, T_{c_{j} k} \phi_{j}\right\rangle\left\langle T_{c_{j} k} \widetilde{\phi}, T_{y} g\right\rangle
$$

is continuous and equals a trigonometric polynomial,

$$
\omega_{j}(y)=\sum_{m \in \mathbb{Z}} c_{m, j} e^{2 \pi i c_{j}^{-1} m y},
$$

where the Fourier coefficients are

$$
c_{m, j}=\frac{1}{c_{j}} \int_{-\infty}^{\infty} \widehat{f}(\gamma) \overline{\widehat{g}\left(\gamma+c_{j}^{-1} m\right)} \overline{\widehat{\phi}_{j}(\gamma)} \widehat{\widetilde{\phi}}_{j}\left(\gamma+c_{j}^{-1} m\right) d \gamma .
$$

Thus, in order to show that the function $\omega$ is continuous it is enough to show that

$$
\sum_{j \in J} \sum_{m \in \mathbb{Z}}\left|c_{m, j}\right|<\infty
$$

this is an easy application of Lemma 3.1. 
The following result measures the deviation from exact reconstruction in terms of the deviation from equality in the duality conditions in (2.6). It generalizes a result from [3]; we discuss this in more detail in Example 3.5.

Theorem 3.3. Assume that the GSI-systems $\left\{T_{c_{j} k} \phi_{j}\right\}_{k \in \mathbb{Z}, j \in J}$ and $\left\{T_{c_{j} k} \widetilde{\phi}_{j}\right\}_{k \in \mathbb{Z}, j \in J}$ are Bessel sequences and satisfy the dual $\alpha$-LIC-condition for all $f \in \mathcal{D}$; denote the associated preframe operators by $T$, resp., $U$. Then

$\left\|I-U T^{*}\right\| \leq\left\|\sum_{j \in J} \frac{1}{c_{j}} \overline{\widehat{\phi}_{j}(\gamma)} \widehat{\widetilde{\phi}}_{j}(\gamma)-1\right\|_{\infty}+\sum_{\alpha \in \Lambda \backslash\{0\}}\left\|\sum_{j \in J_{\alpha}} \frac{1}{c_{j}} \overline{\widehat{\phi}_{j}(\gamma)} \widehat{\widetilde{\phi}}_{j}(\gamma+\alpha)\right\|_{\infty}$

Proof. Note that in terms of the function $\omega$ in (3.2), we have $\omega(0)=\left\langle U T^{*} f, g\right\rangle$. Using (3.3) in Lemma 3.2 with $y=0$ we see that for $f, g \in \mathcal{D}$,

$$
\begin{aligned}
\left\langle\left(U T^{*} f-f\right), g\right\rangle= & \sum_{\alpha \in \Lambda}\left(\int_{-\infty}^{\infty} \widehat{f}(\gamma) \overline{\widehat{g}(\gamma+\alpha)} \sum_{j \in J_{\alpha}} \frac{1}{c_{j}} \overline{\widehat{\phi}_{j}}(\gamma) \widehat{\phi_{j}}(\gamma+\alpha)\right)-\langle\widehat{f}, \widehat{g}\rangle \\
= & \int_{-\infty}^{\infty} \widehat{f}(\gamma) \overline{\widehat{g}(\gamma)}\left(\sum_{j \in J} \frac{1}{c_{j}} \overline{\widehat{\phi}_{j}(\gamma)} \widehat{\widetilde{\phi}_{j}}(\gamma)-1\right) d \gamma \\
& +\sum_{\alpha \in \Lambda \backslash\{0\}} \int_{-\infty}^{\infty} \widehat{f}(\gamma) \overline{\widehat{g}(\gamma+\alpha)} \sum_{j \in J_{\alpha}} \frac{1}{c_{j}} \overline{\widehat{\phi}_{j}(\gamma)} \widehat{\tilde{\phi}_{j}}(\gamma+\alpha) d \gamma .
\end{aligned}
$$

It follows that

$$
\begin{aligned}
\left|\left\langle\left(U T^{*} f-f\right), g\right\rangle\right| \leq & \int_{-\infty}^{\infty}|\widehat{f}(\gamma) \overline{\widehat{g}(\gamma)}|\left|\sum_{j \in J} \frac{1}{c_{j}} \overline{\widehat{\phi}_{j}(\gamma)} \widehat{\widetilde{\phi}}_{j}(\gamma)-1\right| d \gamma \\
& +\sum_{\alpha \in \Lambda \backslash\{0\}} \int_{-\infty}^{\infty}|\widehat{f}(\gamma) \overline{\widehat{g}(\gamma+\alpha)}|\left|\sum_{j \in J_{\alpha}} \frac{1}{c_{j}} \overline{\widehat{\phi}_{j}(\gamma)} \widehat{\tilde{\phi}_{j}}(\gamma+\alpha)\right| d \gamma \\
\leq & \left\|\sum_{j \in J} \frac{1}{c_{j}} \overline{\widehat{\phi}_{j}(\gamma)} \widehat{\widehat{\phi}_{j}}(\gamma)-1||_{\infty}\right\| f\left\|_{2}\right\| g \|_{2} \\
& +\sum_{\alpha \in \Lambda \backslash\{0\}}\left\|\sum_{j \in J_{\alpha}} \frac{1}{c_{j}} \overline{\widehat{\phi}_{j}(\gamma)} \widehat{\widetilde{\phi}}_{j}(\gamma+\alpha)\right\|\left\|_{\infty}\right\| f\left\|_{2}\right\| g \|_{2} .
\end{aligned}
$$

Since $\mathcal{D}$ is dense in $L^{2}(\mathbb{R})$ it follows that

$$
\begin{aligned}
& \left\|U T^{*} f-f\right\|_{2}=\sup _{\|g\|=1}\left|\left\langle\left(U T^{*} f-f\right), g\right\rangle\right| \\
\leq & \left(\left\|\sum_{j \in J} \frac{1}{c_{j}} \overline{\widehat{\phi}_{j}(\gamma)} \widehat{\widetilde{\phi}}_{j}(\gamma)-1\right\|_{\infty}+\sum_{\alpha \in \Lambda \backslash\{0\}}\left\|\sum_{j \in J_{\alpha}} \frac{1}{c_{j}} \overline{\widehat{\phi}_{j}(\gamma)} \widehat{\widetilde{\phi}}_{j}(\gamma+\alpha)\right\|_{\infty}\right)\|f\|_{2},
\end{aligned}
$$

as desired.

We will now derive some consequences of Theorem 3.3. First, let us consider a shift-invariant system, i.e., a collection of functions $\left\{T_{c k} \phi_{j}\right\}_{k \in \mathbb{Z}, j \in J}$, where $c>0$ 
and $\left\{\phi_{j}\right\}_{j \in J}$ is a countable collection of functions in $L^{2}(\mathbb{R})$; this corresponds to a GSI-system where the parameters $c_{j}$ are independent of $j \in J$. The frame analysis of such systems was pioneered by Ron and Shen [19] and Janssen [16]. For a shift-invariant system the index sets $\Lambda$ and $J_{\alpha}$ take the form $\Lambda=c^{-1} \mathbb{Z}, J_{\alpha}=$ $J$. Furthermore, the LIC is automatically satisfied if $\left\{T_{c k} \phi_{j}\right\}_{k \in \mathbb{Z}, j \in J}$ is a Bessel sequence [14]. Thus, we obtain the following explicit version of Theorem 3.3:

Corollary 3.4. Assume that the shift-invariant systems $\left\{T_{c k} \phi_{j}\right\}_{k \in \mathbb{Z}, j \in J}$ and $\left\{T_{c k} \widetilde{\phi}_{j}\right\}_{k \in \mathbb{Z}, j \in J}$ are Bessel sequences and denote the associated preframe operators by $T$, resp., $U$. Then

$$
\begin{aligned}
\left\|I-U T^{*}\right\| \leq & \frac{1}{c}\left(\left\|\sum_{j \in J} \overline{\widehat{\phi}_{j}(\gamma)} \widehat{\widetilde{\phi}}_{j}(\gamma)-c\right\|_{\infty}\right. \\
& \left.+\sum_{n \in \mathbb{Z} \backslash\{0\}}\left\|\sum_{j \in J} \overline{\widehat{\phi}_{j}(\gamma)} \widehat{\tilde{\phi}_{j}}(\gamma+n / c)\right\|_{\infty}\right) .
\end{aligned}
$$

Let us consider a concrete case, namely, the Gabor systems. For $b \in \mathbb{R}$, define the modulation operator on $L^{2}(\mathbb{R})$ by $E_{b} f(x)=e^{2 \pi i b x} f(x)$.

Example 3.5. Given $a, b>0$ the Gabor system generated by a function $g \in$ $L^{2}(\mathbb{R})$ is given by

$$
\left\{E_{m b} T_{n a} g\right\}_{m, n \in \mathbb{Z}}=\left\{e^{2 \pi i m b x} g(x-n a)\right\}_{m, n \in \mathbb{Z}} .
$$

Note that $E_{m b} T_{n a} g(x)=e^{2 \pi i m n a b} T_{n a} E_{m b} g(x)$. It follows that two Gabor frames $\left\{E_{m b} T_{n a} g\right\}_{m, n \in \mathbb{Z}}$ and $\left\{E_{m b} T_{n a} \widetilde{g}\right\}_{m, n \in \mathbb{Z}}$ are dual frames if and only if the shiftinvariant systems $\left\{T_{n a} E_{m b} g\right\}_{m, n \in \mathbb{Z}}$ and $\left\{T_{n a} E_{m b} \widetilde{g}\right\}_{m, n \in \mathbb{Z}}$ are dual frames; furthermore, denoting the preframe operators for $\left\{E_{m b} T_{n a} g\right\}_{m, n \in \mathbb{Z}}$ and $\left\{E_{m b} T_{n a} \widetilde{g}\right\}_{m, n \in \mathbb{Z}}$ by $V$, resp., $W$ and the preframe operators for $\left\{T_{n a} E_{m b} g\right\}_{m, n \in \mathbb{Z}}$ and $\left\{T_{n a} E_{m b} \widetilde{g}\right\}_{m, n \in \mathbb{Z}}$ by $T$, resp., $U$, we have

$$
W V^{*} f=\sum_{m, n \in \mathbb{Z}}\left\langle f, E_{m b} T_{n a} g\right\rangle E_{m b} T_{n a} \widetilde{g}=\sum_{m, n \in \mathbb{Z}}\left\langle f, T_{n a} E_{m b} g\right\rangle T_{n a} E_{m b} \widetilde{g}=U T^{*} f .
$$

Using that $\widehat{E_{m b} g}(\gamma)=T_{m b} \widehat{g}(\gamma)=\widehat{g}(\gamma-b)$, Corollary 3.4 now yields the estimate

$$
\begin{aligned}
\left\|I-W V^{*}\right\| \leq & \frac{1}{a}\left(\left\|\sum_{m \in \mathbb{Z}} \overline{\widehat{g}(\gamma-m b)} \widehat{\widetilde{g}}(\gamma-m b)-a\right\|_{\infty}\right. \\
& \left.+\sum_{n \in \mathbb{Z} \backslash\{0\}}\left\|\sum_{m \in \mathbb{Z}} \overline{\widehat{g}(\gamma-m b)} \widehat{\widetilde{g}}(\gamma-m b-n / a)\right\|_{\infty}\right) .
\end{aligned}
$$

Note that for periodicity reasons it is enough to take the $L^{\infty}$-norm in (3.9) over $\gamma \in[0, b[$. Now it is easy to estimate (3.9), e.g., by imposing certain decay conditions on the functions $g, \widetilde{g}$. In fact, estimates of terms like in (3.9) are standard in frame theory; see, e.g., $[6,7,1]$. 
In the particular case of Gabor frames, a similar result was obtained in the time-domain in the paper [3], however, by applying the stronger conditions that the functions $g, \widetilde{g}$ belong to the Wiener space.

The next example shows that Theorem 3.3 is not appropriate for application to wavelet systems. This will motivate the analysis to follow, which will lead to an alternative method of estimating the deviation from perfect reconstruction.

Example 3.6. Let the scaling operator on $L^{2}(\mathbb{R})$ be given by $D f(x):=2^{1 / 2} f(2 x)$. The wavelet system generated by a function $\psi \in L^{2}(\mathbb{R})$ is the collection of functions

$$
\left\{D^{j} T_{k} \psi\right\}_{j, k \in \mathbb{Z}}=\left\{2^{j / 2} \psi\left(2^{j} x-k\right)\right\}_{j, k \in \mathbb{Z}}
$$

A wavelet system $\left\{D^{j} T_{k} \psi\right\}_{j, k \in \mathbb{Z}}$ in $L^{2}(\mathbb{R})$ is a GSI-system. In fact,

$$
\left\{D^{j} T_{k} \psi\right\}_{j, k \in \mathbb{Z}}=\left\{T_{2^{-j} k} D^{j} \psi\right\}_{j, k \in \mathbb{Z}}=\left\{T_{c_{j} k} \phi_{j}\right\}_{k \in \mathbb{Z}, j \in J},
$$

where $c_{j}=2^{-j}, \phi_{j}=D^{j} \psi, J=\mathbb{Z}$. Note that the set $\Lambda$ in (2.4) can be written as

$$
\Lambda=\left\{2^{j} n \mid j, n \in \mathbb{Z}\right\}=\left\{2^{k} m \mid k \in \mathbb{Z}, \operatorname{modd}\right\}
$$

and, given $\alpha \in \Lambda$ on the form $\alpha=2^{k} m$ where $k \in \mathbb{Z}$ and $m$ is odd,

$$
J_{\alpha}=\left\{j \in \mathbb{Z} \mid \exists n \in \mathbb{Z} \text { such that } 2^{k} m=2^{j} n\right\}=\{\ldots, k-1, k\} .
$$

The duality equations (2.7) take the form

$$
\left\{\begin{array}{c}
\sum_{j \in \mathbb{Z}} \widehat{\widehat{\psi}\left(2^{-j} \gamma\right)} \widehat{\widetilde{\psi}}\left(2^{-j} \gamma\right)-1=0 \\
\sum_{j=-\infty}^{k} \overline{\widehat{\psi}\left(2^{-j} \gamma\right)} \widehat{\widetilde{\psi}}\left(2^{-j}\left(\gamma+2^{k} m\right)\right)=0, k \in \mathbb{Z}, m \text { odd }
\end{array}\right.
$$

For $m, k \in \mathbb{Z}$, consider the function

$$
\theta_{m, k}(\gamma):=\sum_{j=-\infty}^{k} \overline{\widehat{\psi}\left(2^{-j} \gamma\right)} \widehat{\widetilde{\psi}}\left(2^{-j}\left(\gamma+2^{k} m\right)\right)
$$

then

$$
\begin{aligned}
\theta_{m, k+1}(\gamma) & =\sum_{j=-\infty}^{k+1} \overline{\widehat{\psi}\left(2^{-j} \gamma\right)} \widehat{\widetilde{\psi}}\left(2^{-j}\left(\gamma+2^{k+1} m\right)\right) \\
& =\sum_{j=-\infty}^{k+1} \overline{\widehat{\psi}\left(2^{-(j-1)} \gamma / 2\right)} \widehat{\widetilde{\psi}}\left(2^{-(j-1)}\left(\gamma / 2+2^{k} m\right)\right) \\
& =\sum_{j=-\infty}^{k} \overline{\widehat{\psi}\left(2^{-j} \gamma / 2\right)} \widehat{\widetilde{\psi}}\left(2^{-j}\left(\gamma / 2+2^{k} m\right)\right)=\theta_{m, k}(\gamma / 2)
\end{aligned}
$$


In particular, this shows that $\left\|\theta_{m, k}\right\|_{\infty}$ is independent of $k \in \mathbb{Z}$; in other words, the second set of equations in (3.11) holds if and only if the equation holds for $k=0$, i.e., if and only if

$$
\sum_{j=-\infty}^{0} \overline{\widehat{\psi}\left(2^{-j} \gamma\right)} \widehat{\widetilde{\psi}}\left(2^{-j}(\gamma+m)\right)=0, \forall m \text { odd }
$$

If just one of the $\alpha$-equations in (2.7) do not hold, then $\left\|\theta_{m, k}\right\|_{\infty} \neq 0$ for some $m, k$; since this term appears infinitely often in the infinite sum on the righthand-side of (3.7) the estimate is not useful. The conclusion is that for wavelet systems the deviation from equality in the duality equations seldom give a useful estimate for the deviation from perfect reconstruction.

Motivated by the negative outcome in Example 3.10 we will now derive an alternative method for estimating the deviation from perfect reconstruction. Here we consider again two GSI-systems $\left\{T_{c_{j} k} \phi_{j}\right\}_{k \in \mathbb{Z}, j \in J}$ and $\left\{T_{c_{j} k} \widetilde{\phi}_{j}\right\}_{k \in \mathbb{Z}, j \in J}$, but now we will measure the deviation from perfect reconstruction in terms of how much the two systems deviate from a pair of dual frames $\left\{T_{c_{j} k} g_{j}\right\}_{k \in \mathbb{Z}, j \in J}$ and $\left\{T_{c_{j} k} \widetilde{g}_{j}\right\}_{k \in \mathbb{Z}, j \in J}$, measured via the Bessel condition in Lemma 2.2:

Theorem 3.7. Assume that the GSI-systems $\left\{T_{c_{j} k} \phi_{j}\right\}_{k \in \mathbb{Z}, j \in J}$ and $\left\{T_{c_{j} k} \widetilde{\phi}_{j}\right\}_{k \in \mathbb{Z}, j \in J}$ are Bessel sequences, with preframe operators $T$ and $U$. Furthermore, let $\left\{T_{c_{j} k} g_{j}\right\}_{k \in \mathbb{Z}, j \in J}$ and $\left\{T_{c_{j} k} \widetilde{g}_{j}\right\}_{k \in \mathbb{Z}, j \in J}$ be a pair of dual frames for $L^{2}(\mathbb{R})$, with Bessel bounds $B_{g}$, respectively, $B_{\widetilde{g}}$. Finally, let

$$
B_{g-\phi}:=\sup _{\gamma \in \mathbb{R}} \sum_{j \in J} \sum_{k \in \mathbb{Z}} \frac{1}{c_{j}}\left|\left(\widehat{g}_{j}-\widehat{\phi}_{j}\right)(\gamma)\left(\widehat{g_{j}}-\widehat{\phi}_{j}\right)\left(\gamma-c_{j}^{-1} k\right)\right|
$$

and

$$
B_{\widetilde{g}-\widetilde{\phi}}:=\sup _{\gamma \in \mathbb{R}} \sum_{j \in J} \sum_{k \in \mathbb{Z}} \frac{1}{c_{j}}\left|\left(\widehat{\widetilde{g}}_{j}-\widehat{\widetilde{\phi}}_{j}\right)(\gamma)\left(\widehat{\widetilde{g}}_{j}-\widehat{\widetilde{\phi}}_{j}\right)\left(\gamma-c_{j}^{-1} k\right)\right|
$$

Then

$$
\left\|I-U T^{*}\right\| \leq B_{\widetilde{g}}^{1 / 2} B_{g-\phi}^{1 / 2}+B_{\widetilde{g}-\widetilde{\phi}}^{1 / 2}\left(B_{g-\phi}^{1 / 2}+B_{g}^{1 / 2}\right) .
$$

Proof. Denote the preframe operators for $\left\{T_{c_{j} k} g_{j}\right\}_{k \in \mathbb{Z}, j \in J}$ and $\left\{T_{c_{j} k} \widetilde{g}_{j}\right\}_{k \in \mathbb{Z}, j \in J}$ by $V$ and $W$, respectively. Then for $f \in L^{2}(\mathbb{R})$,

$$
\begin{aligned}
\left\|f-U T^{*} f\right\| & =\left\|W V^{*} f-U T^{*} f\right\| \\
& =\left\|W\left(V^{*}-T^{*}\right) f+(W-U) T^{*} f\right\| \\
& \leq(\|W\|\|V-T\|+\|W-U\|\|T\|)\|f\|
\end{aligned}
$$

We now estimate the terms in (3.13). Clearly $\|W\| \leq B_{\widetilde{g}}^{1 / 2}$. Furthermore, $V-T$ is the preframe operator for the GTI-system $\left\{T_{c_{j} k}\left(g_{j}-\phi_{j}\right)\right\}_{k \in \mathbb{Z}, j \in J}$; thus Lemma 2.2 implies that $\|V-T\| \leq B_{g-\phi}^{1 / 2}$. Similarly, $\|W-U\| \leq B_{\widetilde{g}-\widetilde{\phi}}^{1 / 2}$. Using that $\|T\| \leq\|T-V\|+\|V\| \leq B_{g-\phi}^{1 / 2}+B_{g}^{1 / 2}$ we finally arrive at the estimate (3.12). 
Theorem 3.7 has has recently been used to construct approximately dual frames of Gabor frames generated by the Gaussian; see [2] for details. A further consequence of the analysis in [2] is that certain scalings of the B-splines $B_{N}$ converge towards the Gaussian whenever $N \rightarrow \infty$, in the sense that the Bessel bound for any Gabor system generated by the difference between the scaled B-splines and the Gaussian tend to zero. In particular the result implies that for any choice of translation parameter $a>0$ and modulation parameter $b>0$ such that $a b<1$, the Gabor system generated by the scaled B-splines generate frames whenever the order of the B-spline is sufficiently high. This result is rather surprising in view of the many known obstructions to the frame property for B-splines, see $[8,12,11,17,15]$. We also note that the arguments used in the proof are of a general nature, which allows for a similar formulation for general frames in Hilbert spaces [5].

The next result is a consequence of Theorem 3.7 and its proof. Actually the result highlights the key idea behind all the results in the paper. Indeed we will consider two dual GSI frames $\left\{T_{c_{j} k} g_{j}\right\}_{k \in \mathbb{Z}, j \in J}$ and $\left\{T_{c_{j} k} \widetilde{g}_{j}\right\}_{k \in \mathbb{Z}, j \in J}$ and estimate the deviation from perfect reconstruction that occur when the windows - typically due to practical constraints - are "truncated." Due to the nature of the GSI-conditions the truncation will take place in the Fourier domain:

Corollary 3.8. Assume that $\left\{T_{c_{j} k} g_{j}\right\}_{k \in \mathbb{Z}, j \in J}$ and $\left\{T_{c_{j} k} \widetilde{g}_{j}\right\}_{k \in \mathbb{Z}, j \in J}$ are dual frames, and that

$$
B_{g}:=\sup _{\gamma \in \mathbb{R}} \sum_{j \in J} \sum_{k \in \mathbb{Z}} \frac{1}{c_{j}}\left|\widehat{g}_{j}(\gamma) \widehat{g}_{j}\left(\gamma-c_{j}^{-1} k\right)\right|<\infty
$$

and

$$
B_{\widetilde{g}}:=\sup _{\gamma \in \mathbb{R}} \sum_{j \in J} \sum_{k \in \mathbb{Z}} \frac{1}{c_{j}}\left|\widehat{\widetilde{g}}_{j}(\gamma) \widehat{\widetilde{g}}_{j}\left(\gamma-c_{j}^{-1} k\right)\right|<\infty .
$$

Given two collections of compact sets $\left\{S_{j}\right\}_{j \in J},\left\{\widetilde{S}_{j}\right\}_{j \in J} \subset \mathbb{R}$, define the functions $\phi_{j}$ and $\widetilde{\phi}_{j}$ by

$$
\widehat{\phi}_{j}:=\widehat{g}_{j} \chi_{S_{j}}, \quad \widehat{\widetilde{\phi}}_{j}:=\widehat{\widetilde{g}}_{\gamma_{\widetilde{S_{j}}}}, j \in J .
$$

Finally, denote the preframe operators for the GSI systems $\left\{T_{c_{j} k} \phi_{j}\right\}_{k \in \mathbb{Z}, j \in J}$ and $\left\{T_{c_{j} k} \widetilde{\phi}_{j}\right\}_{k \in \mathbb{Z}, j \in J}$ by $T$, respectively $U$. Then

$$
\begin{aligned}
\left\|I-U T^{*}\right\| \leq & B_{\widetilde{g}}^{1 / 2}\left(\sup _{\gamma \in \mathbb{R}} \sum_{j \in J} \sum_{k \in \mathbb{Z}} \frac{1}{c_{j}}\left|\left(\widehat{g_{j}} \chi_{\mathbb{R} \backslash S_{j}}\right)(\gamma)\left(\widehat{g_{j}} \chi_{\mathbb{R} \backslash S_{j}}\right)\left(\gamma-c_{j}^{-1} k\right)\right|\right)^{1 / 2} \\
& +B_{g}^{1 / 2}\left(\sup _{\gamma \in \mathbb{R}} \sum_{j \in J} \sum_{k \in \mathbb{Z}} \frac{1}{c_{j}}\left|\left(\widehat{\widetilde{g}_{j}} \chi_{\mathbb{R} \backslash \widetilde{S_{j}}}\right)(\gamma)\left(\widehat{\widetilde{g}_{j}} \chi_{\mathbb{R} \backslash \widetilde{S_{j}}}\right)\left(\gamma-c_{j}^{-1} k\right)\right|\right)^{1 / 2} .
\end{aligned}
$$

Proof. The definition of the functions $\phi_{j}$ immediately show that

$$
\sum_{j \in J} \sum_{k \in \mathbb{Z}} \frac{1}{c_{j}}\left|\widehat{\phi}_{j}(\gamma) \widehat{\phi}_{j}\left(\gamma-c_{j}^{-1} k\right)\right| \leq \sum_{j \in J} \sum_{k \in \mathbb{Z}} \frac{1}{c_{j}}\left|\widehat{g}_{j}(\gamma) \widehat{g}_{j}\left(\gamma-c_{j}^{-1} k\right)\right| \leq B_{g}
$$


for a.e. $\gamma \in \mathbb{R}$; thus $\left\{T_{c_{j} k} \phi_{j}\right\}_{k \in \mathbb{Z}, j \in J}$ is a Bessel sequence. By the symmetry in the conditions, also $\left\{T_{c_{j} k} \widetilde{\phi}_{j}\right\}_{k \in \mathbb{Z}, j \in J}$ is a Bessel sequence.

In order to obtain the desired estimate on $\left\|I-U T^{*}\right\|$ we now refer to (3.13). As before, $\|W\| \leq B_{\widetilde{g}}^{1 / 2}$. Furthermore, by (3.15), $B_{g}$ is a Bessel bound for $\left\{T_{c_{j} k} \phi_{j}\right\}_{k \in \mathbb{Z}, j \in J}$, so $\|T\| \leq B_{g}$. The rest follows from the estimates on $\|V-T\|$ and $\|W-U\|$ in the proof of Theorem 3.7.

It is clear from the proof of Corollary 3.8 that the same idea can be used "the opposite way around." That is, if we know that certain GSI-system generated by compactly supported truncated versions $g_{j}, \widetilde{g_{j}}$ of some functions $\varphi_{j}, \widetilde{\varphi_{j}}$ generate dual frames or approximately dual frames, then Bessel estimates will yield information about how far the GSI-systems generated by $\varphi_{j}, \widetilde{\varphi_{j}}$ are from yielding perfect reconstruction. We leave the derivations of concrete statements to the interested reader.

In the concrete case of wavelet systems we will now derive a completely explicit version of Corollary 3.8. All terms will be formulated via Bessel conditions that can be estimated by standard techniques in frame theory $[6,7,1]$. We will see that it is important that the "cut-of" determined by the sets $S_{j}$ in Corollary 3.8 is allowed to depend on $j$ :

Example 3.9. Consider a dual pair of frames $\left\{D^{j} T_{k} g\right\}_{j, k \in \mathbb{Z}},\left\{D^{j} T_{k} \widetilde{g}\right\}_{j, k \in \mathbb{Z}}$; as we have seen in Example 3.6 they correspond to GSI-systems $\left\{T_{c_{j} k} g_{j}\right\}_{k \in \mathbb{Z}, j \in J}$ and $\left\{T_{c_{j} k} \widetilde{g}_{j}\right\}_{k \in \mathbb{Z}, j \in J}$ with $c_{j}=2^{-j}, g_{j}=D^{j} g, \widetilde{g_{j}}=D^{j} \widetilde{g}$, and $J=\mathbb{Z}$. Now,

$$
\widehat{g_{j}}(\gamma)=D^{-j} \widehat{g}(\gamma)
$$

thus, it is natural to consider the functions $\phi_{j}$ defined by

$$
\left.\widehat{\phi}_{j}(\gamma)=D^{-j}\left(\widehat{g} \chi_{[-N, N]}\right)(\gamma)=\widehat{g}_{j}(\gamma) \chi_{\left[-2^{j} N, 2^{j} N\right]}\right)(\gamma)
$$

for some $N \in \mathbb{N}$; this corresponds exactly to (3.14) with $S_{j}=\left[-2^{j} N, 2^{j} N\right]$. Alternatively, denoting the Fourier transform by $\mathcal{F}$ and using the convolution, to be denoted by $*$,

$$
\phi_{j}=\mathcal{F}^{-1} D^{-j}\left(\widehat{g} \chi_{[-N, N]}\right)=D^{j} \mathcal{F}^{-1}\left(\widehat{g} \chi_{[-N, N]}\right)=D^{j}\left(g * \mathcal{F}^{-1} \chi_{[-N, N]}\right) ;
$$

thus, the GSI-system $\left\{T_{c_{j} k} \phi_{j}\right\}_{k \in \mathbb{Z}, j \in J}$ in fact equals the wavelet system $\left\{D^{j} T_{k} \phi\right\}_{j, k \in \mathbb{Z}}$ with $\phi=g * \mathcal{F}^{-1} \chi_{[-N, N]}$.

Clearly, we also define the function $\widetilde{\phi}$ by $\widetilde{\phi}=\widetilde{g} * \mathcal{F}^{-1} \chi_{[-N, N]}$. Now, letting $T$ and $U$ denote the preframe operators for the wavelet systems $\left\{D^{j} T_{k} \phi\right\}_{j, k \in \mathbb{Z}}$ and $\left\{D^{j} T_{k} \widetilde{\phi}\right\}_{j, k \in \mathbb{Z}}$, the estimate in Example 3.6 takes the form

$$
\begin{aligned}
\left\|I-U T^{*}\right\| & \leq \\
& B_{\widetilde{g}}^{1 / 2}\left(\sup _{\gamma \in[1,2]} \sum_{j \in \mathbb{Z}} \sum_{k \in \mathbb{Z}}\left|\left(\widehat{g} \chi_{\mathbb{R} \backslash[-N, N]}\right)\left(2^{-j} \gamma\right)\left(\widehat{g} \chi_{\mathbb{R} \backslash[-N, N]}\right)\left(2^{-j} \gamma-k\right)\right|\right)^{1 / 2} \\
+ & B_{g}^{1 / 2}\left(\sup _{\gamma \in[1,2]} \sum_{j \in \mathbb{Z}} \sum_{k \in \mathbb{Z}}\left|\left(\widehat{\widetilde{g}} \chi_{\mathbb{R} \backslash[-N, N]}\right)\left(2^{-j} \gamma\right)\left(\widehat{\widetilde{g}} \chi_{\mathbb{R} \backslash[-N, N]}\right)\left(2^{-j} \gamma-k\right)\right|\right)^{1 / 2}
\end{aligned}
$$


The terms appearing in the parentheses can be estimated exactly as in the standard calculations for the Bessel bound of a wavelet system, see $[6,7,1]$.

Grants. Research partially supported by Consejo Nacional de Investigaciones Científicas y Técnicas (CONICET) and Universidad Nacional de San Luis (UNSL) with grants PIP 11220110100033CO and PROICO 317902, respectively. Ole Christensen would like to thank CONICET and Department of Mathematics, University of San Luis, for support and hospitality during visits in 2015 and 2016. We would also like to thank the reviewer for useful suggestions for improvements, especially for prompting us to add more comments about applications.

\section{REFERENCES}

1. Christensen, O.: An introduction to frames and Riesz bases. Second expanded edition. Birkhäuser (2016)

2. Christensen, O., Kim, H. O., and Kim, R. Y.: B-spline approximations of the Gaussian and their frame properties. Preprint, 2017.

3. Christensen, O. and Laugesen, R.: Approximately dual frames in Hilbert spaces and applications to Gabor frames. Sampling Theory in Signal and Image Processing 9 (2011), 77-90.

4. Christensen, O. and Rahimi, A.: Frame properties of wave packet systems in $L^{2}\left(R^{d}\right)$. Adv. Comp. Math. 29 no. 2 (2008), 101-111.

5. Christensen, O., and Zakowicz, M.: Paley-Wiener type perturbations of frames and the deviation from perfect reconstruction. Azerbaijan J. Math., to appear 2017.

6. Daubechies, I.: The wavelet transformation, time-frequency localization and signal analysis. IEEE Trans. Inform. Theory 36 (1990), 961-1005.

7. Daubechies, I.: Ten lectures on wavelets. SIAM, Philadelphia, 1992.

8. Del Prete, V.: Estimates, decay properties, and computation of the dual function for Gabor frames. J. Fourier Anal. Appl. 5 (1999), 545-562.

9. Feichtinger, H. G., Grybos, A., and Onchis, D. M.: Approximate dual Gabor atoms via the adjoint lattice method. Adv. Comp. Math. 40 (2014), 651-665.

10. Feichtinger, H. G., Onchis, D. M., and Wiesmeyr, C.: Construction of approximate dual wavelet frames. Adv. Comp. Math. 40 (2014), 273-282.

11. Gröchenig, K.: The mystery of Gabor frames. J. Fourier Anal. Appl. 20 (2014), 865-895.

12. Gröchenig, K., Janssen, A. J. E. M., Kaiblinger, N., and Pfander, G.: Note on B-splines, wavelet scaling functions, and Gabor frames. IEEE Trans. Inf. Theory 49 no. 12 (2003), 3318-3320.

13. Hernandez, E., Labate, D., and Weiss, G.: A unified characterization of reproducing systems generated by a finite family II. J. Geom. Anal. 12 no. 4 (2002), 615-662.

14. Jakobsen, M. S., and Lemvig, J.: Reproducing formulas for generalized translation invariant systems on locally compact groups. Trans. Amer. Math. Soc., to appear 2015.

15. Lemvig, J., and Nielsen, K. H.: A counterexample to the B-spline conjecture for Gabor frames. Preprint, 2015.

16. Janssen, A.J.E.M.: The duality condition for Weyl-Heisenberg frames. In "Gabor analysis: theory and application", (eds. Feichtinger, H. G. and Strohmer, T.). Birkhäuser, Boston, 1998.

17. Kloos, T., and Stöckler, J.: Zak transforms and Gabor frames of totally positive functions and exponential B-splines. J. Approx. Theory 184 (2014), 209-237.

18. Labate, D. Weiss, G and Wilson, E.: An Approach to the Study of Wave Packet systems.Contemp. Mathematics, Wavelets, Frames and Operator Theory, 345, (2004), pp.215235 . 
GENERALIZED SHIFT-INVARIANT SYSTEMS AND APPROXIMATELY DUAL FRAMES 13

19. Ron, A. and Shen, Z.: Frames and stable bases for shift-invariant subspaces of $L^{2}\left(\mathbb{R}^{d}\right)$. Canad. J. Math. 47 no. 5 (1995), 1051-1094.

20. Ron, A. and Shen, Z.: Generalized shift-invariant systems. Const. Appr. 22 no. 1 (2005), pp. $1-45$.

${ }^{1}$ Instituto de Matemtica Aplicada San Luis, imasl. Universidad Nacional de San Luis and CONICET. D5700HHW San Luis. Argentina.

E-mail address: abenaven@unsl.edu.ar

${ }^{2}$ Technical University of Denmark, DTU Compute, 2800 Lyngby, Denmark

E-mail address: ochr@dtu.dk

${ }^{3}$ Departamento de Matemática, Universidad Nacional de San Luis, D5700HhW San Luis, Argentina.

E-mail address: mzakowi@unsl.edu.ar 